\title{
Importancia de la Innovación en el contexto académico, tecnológico y en el desarrollo regional
}

\author{
Gallego Tavera, Sindy-Yuliana ${ }^{1,2(*)}$; Polo-Salcedo, Ana Lucila'; Gómez-Ospina, Paola Andrea²
}

'Vicerrectoría de Investigaciones, Grupo GORAS, Universidad Católica Luis Amigó, Facultad de psicología Medellín, Colombia

${ }^{2}$ Universidad de Medellín, Facultad de Derecho, Medellín, Colombia

\section{RESUMEN}

En este manuscrito se presentan una serie de apartados en los que la innovación demuestra ser el motor para generar el progreso y la calidad de vida de las naciones más desarrolladas y de aquellas que están en vías de desarrollo. En un primer apartado, se explicará cómo la innovación impacta a diferentes naciones del mundo. En un segundo apartado, se abordará el desarrollo de la tecnología como instrumento para el crecimiento de la economía a través de los datos de posicionamiento móvil. En un tercer apartado, se tocará la promoción de microempresas para el desarrollo económico regional. En un cuarto apartado, se desarrollará una temática concerniente a la inversión extranjera como herramienta del desarrollo regional. En un quinto apartado, se expondrá lo relativo al fenómeno de la globalización como reemplazo de la economía tradicional. En un sexto apartado, se elucidará el papel que tienen las redes sociales en el contexto de desarrollo global. En un séptimo apartado, se presentarán los efectos de la presión global sobre salarios y empleo. En un octavo apartado, se exhibirán las consecuencias del trabajo infantil y género. En un noveno apartado, se tocarán fenómenos económicos y sociales de suma relevancia como lo son la globalización y migración. En un décimo apartado, se cerrará el capítulo con las conclusiones, las cuales presentan las implicaciones y limitaciones de las políticas nacionales e internacionales que atañen a la innovación.

Palabras clave: innovación; tecnología; contexto; globalización; desarrollo.

\section{Importance of Innovation in the Academic and Technological Context and in Regional Development}

\section{Abstract}

This manuscript presents a series of sections in which innovation proves to be the engine to generate progress and the quality of life of the most developed nations and those that are developing. In a first section, it will be explained how innovation impacts different nations of the world. In a second section, the development of technology as an instrument for the growth of the economy will be tackled through mobile positioning data. In a third section, the promotion of micro-enterprises for regional economic development will be discussed. In a fourth section, a subject matter will be developed concerning foreign investment as a tool for regional development. In a fifth section, we will discuss the phenomenon of globalization as a replacement for the traditional economy. In a sixth section, the role of social networks in the context of global development will be elucidated. In a seventh section, the effects of global pressure on wages and employment will be presented. In an eighth section, the consequences of child labor and gender will be shown. In a ninth section, highly relevant economic and social phenomena such as globalization and migration will be discussed. In a tenth section, the chapter will be closed with the conclusions, which present the implications and limitations of national and international policies regarding innovation.

Keywords: innovation; technology; context; globalization; development. 


\section{INTRODUCCIÓN}

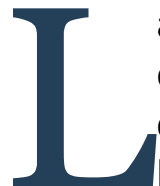

a innovación juega un papel decisivo en la evolución de las sociedades humanas, por cuanto se requieren no solo de avances en las distintas disciplinas de las ciencias exactas, naturales, sociales y humanas, sino que es indispensable que se generen políticas de cooperación internacional, entendiendo el conocimiento humano como un bien universal que debe traspasar las barreras transnacionales.

En este manuscrito se presentan una serie de apartados en los que la innovación demuestra ser el motor para generar el progreso y la calidad de vida de las naciones más desarrolladas y de aquellas que están en vías de desarrollo. En un primer apartado, se explicará cómo la innovación impacta a diferentes naciones del mundo. En un segundo apartado, se abordará el desarrollo de la tecnología como instrumento para el crecimiento de la economía a través de los datos de posicionamiento móvil. En un tercer apartado, se comentará la promoción de microempresas para el desarrollo económico regional. En un cuarto apartado, se desarrollará una temática concerniente a la inversión extranjera como herramienta del desarrollo regional. En un quinto apartado, se expondrá lo relativo al fenómeno de la globalización como reemplazo de la economía tradicional. En un sexto apartado, se dilucida el papel que tienen las redes sociales en el contexto de desarrollo global. En un séptimo apartado, se presentarán los efectos de la presión global sobre salarios y empleo. En un octavo apartado, se exhibirán las consecuencias del trabajo infantil y género. En un noveno apartado, se abordarán fenómenos económicos y sociales de suma relevancia como lo son la globalización y migración. Finalmente, se proporcionarán las conclusiones del conglomerado de apartados.

\section{MARCO TEÓRICO}

En el contexto educativo internacional, la innovación ha sido comprendida como un elemento inherente en el desarrollo económico y social. El gobierno de Malasia, por ejemplo, con el fin de incrementar los niveles económicos, se comprometió con las universidades para promover iniciativas que estimularan el crecimiento de las actividades de innovación, permitiendo que se generen investigaciones relacionadas con la innovación en todos los campos. Esto último con la participación e interacción del estado con políticas públicas que benefician el crecimiento de las universidades, lo que les ha permitido transformarse hacia un modelo más competitivo y reconocido internacionalmente. Además, como consecuencia del reconocimiento y la renovación de estrategias alentadoras, algunas de las universidades en Malasia no solo han posicionado con éxito sus productos de investigación en el mercado, si no también, han generado una estrategia efectiva para elaborar sus propias patentes y productos generando de este modo ingresos mientras contribuyen al desarrollo económico del estado (Reid y Baker, 2018).

Por otro lado, el gobierno de los Estados Unidos les permitió a las universidades patentar sus investigaciones con los fondos federales a través de la Ley de Bayh-Dole de 1980, lo cual fue seguido por algunas universidades en todo el mundo.

La importancia de la propiedad intelectual se ha extrapolado a diferentes industrias, donde se han implementado estrategias para fortalecer la protección de la misma, en las que el objetivo principal está en la construcción, conservación, innovación y producción.

Como consecuencia de la tensión entre innovación y la propiedad intelectual, se ha generado la necesidad de crear políticas públicas claras que no interfieran con el proceso de innovación en las universidades o las diferentes industrias que participan en el desarrollo del crecimiento económico del país con estrategias de innovación vinculadas a investigaciones rigurosas.

Cabe mencionar que el desarrollo de la innovación tecnológica, se presenta en la actualidad como una alternativa que permitiría automatizar los procesos de aprendizaje disminuyendo recursos económicos a largo plazo. A continuación, tomaremos como ejemplo el caso del posicionamiento móvil. (Raduege, 2018)

\section{Desarrollo de la tecnología como instrumento para el crecimiento de la economía a través de los datos de posicionamiento móvil}

La tecnología móvil; es una fuente de información que ha sido de gran ayuda para extraer datos estadísticos, los cuales son utilizados para valorar el crecimiento económico de las regiones. Por esta razón la necesidad de que los datos ofrecidos sean reales y confiables; teniendo en cuenta esto, los métodos y datos estadísticos proporcionados por la tecnología 
móvil deben ser reconocidos internacionalmente. Sin embargo, por motivos de confidencialidad e inversión financiera, este recurso no está disponible para todos los casos, aunque la automatización de los procedimientos reduce significativamente el costo económico y la inversión del tiempo a largo plazo. Por otro lado, coexiste la limitación con respecto a la protección de datos del cliente, debido a que el acceso otorgado no cuenta con su consentimiento la mayoría de las veces (Novack, Hong, Dixon y Granpeesheh, 2018).

En consecuencia, el número de llamadas telefónicas ha sido usado con el fin de establecer indicadores sobre la actividad económica de una región, para corroborar patrones en la actividad de las llamadas. Se analizan en detalle áreas residenciales o comerciales, en las que se tiene en cuenta el uso del territorio, garantías públicas como seguridad, transporte, economía, entre otros. Por consiguiente, el grupo de aplicaciones para los datos móviles involucra el análisis en las diferentes áreas residenciales o comerciales mencionadas.

Haciendo mención de un caso particular, los indicadores del transporte público proporcionan información confiable para que sea posible tomar decisiones y mejorar la planificación de la red (zonas concurridas, zonas más concurridas según temporalidad, densidad poblacional, entre otros). De la misma manera, los patrones de actividad móvil permiten intervenciones en tiempo real en función de seguridad pública, pese a que hasta ahora hay pocos ejemplos a nivel internacional de que estos datos sean usados en función del desarrollo urbano.

Es importante mencionar que la universidad de Letonia dispone de la red móvil más grande de dicho país "Latvijas Mobilais Telefons" (LMT), la cual se ha posicionado como líder en cuanto a innovación, desarrollo sostenible y competitividad en el mercado de Letonia y en la región del Báltico. (Hagger y Weed, 2018).

Por otra parte, si bien la tecnología móvil ha sido usada para el análisis de indicadores económicos, indicadores de transporte público y patrones de actividades en zonas comerciales y residenciales; actualmente su uso se ha ampliado hacia diferentes áreas de gran impacto como la seguridad, particularmente de las mujeres.

Para explorar con más detalle lo que significa el uso de la tecnología móvil en relación con la seguridad de las mujeres, tomaremos como referencia el uso del GPS y la comunicación móvil para el modelo de seguridad de las mujeres en India. Hasta ahora, el uso de la tecnología GPS como sistema de ubicación global ha incrementado su popularidad como consecuencia de sus múltiples áreas de aplicación; como los sistemas de navegación, mapas y herramientas de rastreo. Particularmente en India, en los últimos años la tasa de criminalidad hacia las mujeres ha aumentado considerablemente, y en conjunto, las acciones referentes a la agresión sexual, violación sexual y violación doméstica. Es por esto que, el servicio del GPS Women Safety Systems (WSS) se ha convertido en una de las aplicaciones más necesarias y cautivadoras para las usuarias móviles en los últimos tiempos. No obstante, el uso del WSS genera algunos problemas considerables en aspectos tales como, la calidad en el servicio, facilidad del uso, gasto de energía y el seguimiento de navegación (Bauleo, Van y Radon, 2018).

El gobierno de la India ha optado por una propuesta rentable y de fácil acceso para implementar la seguridad y protección de sus habitantes, aun cuando en dicho sistema se dispone de móviles con GPS de alto costo y su valor no ha sido de fácil acceso para la población de clase media y baja de la India. (Bauleo y cols., 2018).

A pesar de ello, actualmente el uso y acceso de teléfonos móviles ha logrado sus niveles más elevados. Un ejemplo claro de ello es la encuesta realizada por la Competition Comission of India (CCI) en 2013 informó que actualmente 897.02 millones de usuarios utilizan teléfonos móviles en la India y 352.47 millones son mujeres. Esto quiere decir que, más del $50 \%$ de las mujeres en India usan teléfonos móviles, teniendo en cuenta que en el futuro esta cifra probablemente sería mayor. Por otro lado, la encuesta realizada por el Institute of Objective en 2011 de Nueva Delhi, India, ha expuesto que la tasa de criminalidad de las mujeres se ha sostenido continuamente desde el año 2000 hasta el 2011 mostrando menor incidencia. Estos datos han influenciado las propuestas para solucionar el problema de inseguridad en la mujer a través de un sistema especial y como resultado, el Women Safety Systems (WSS) se ha convertido en una herramienta fundamental por más de una década. (Sis-Çelik y Kırca, 2018).

En la presentación de los algoritmos novedosos que propone el nuevo sistema del WSS ha sido fundamental la llamada para alarma de emergencia (CEA); la funcionalidad de esta tecnología consiste en que cuando las mujeres perciben una circunstancia peligrosa pueden presionar un botón que activará la ruta de llamada de emergencia, la cual posterior- 
mente enviará la información de los datos de su ubicación exacta a la estación de policía local y a la ambulancia. Esto se logra a través de el botón PGPSB que tiene la función de activar un receptor de GPS móvil y los puntos de paso se comunicarían con el CWSGIS en forma de mandar WPS constantemente. Además, este sistema cuenta con un procedimiento diseñado para evitar el mal uso de la tecla PGPSB; cuya activación de este sistema se produce a través de dos teclas previamente configuradas. El PGPSB solo será llamado por el procesador móvil si luego de presionar la primera tecla configurada, la segunda se mantiene presionada durante 10 segundos. En la configuración inicial las teclas programadas son el número " 0 ", aunque posteriormente el usuario podrá cambiarlas como lo desee. Por otro lado, incluso si el teléfono del usuario se apaga en medio de la situación peligrosa, pero ha presionado el mecanismo CEA, éste funcionará con la asistencia de la batería del BIOS.

Si bien la propuesta para las mujeres que disponen de teléfonos móviles se ha diseñado con la intención de presentar una solución para la inseguridad. Hasta ahora, la seguridad para el gobierno de la India se continúa expresando como un desafío. En resumen, como consecuencia de la falta de recursos y estrategias que permitan a las diferentes regiones o departamentos acceder al análisis de los datos estadísticos de posicionamiento móvil, se presenta como propuesta el análisis de iniciativas políticas para impulsar el desarrollo regional (Tang, Ghose, Hoque, Hao, y Yaya, 2019).

\section{Promoción de microempresas para el desarrollo económico regional}

El desarrollo de las microempresas ha sido presentado a nivel mundial como una propuesta en la intervención del desempleo. Los intentos de los diferentes gobiernos por impulsar esta iniciativa se fundamentan en promover el crecimiento de la economía a nivel regional y del estado. Como consecuencia, las microempresas son promotoras de la innovación, a menudo surgen como resultado del intercambio de ideas conformadas por familiares o personas cercanas. Esto quiere decir que sus operaciones se restringen al territorio local y la limitación económica puede ser un problema recurrente, aunque también pueden pertenecer a una franquicia. Normalmente, quienes dirigen la compañía no cuentan con la formación requerida en habilidades de administración, mercadeo o recursos económicos y humanos. Son empresas independientes vinculadas íntimamente con el espíritu empresarial y la innovación. Estos elementos no sólo son característicos de las microempresas, sino que a menudo representan un problema para el desarrollo regional, debido a la decisión deliberada de mantener la compañía pequeña porque los propietarios aprecian el equilibrio entre los negocios y un estilo de vida tranquilo (O'Neill y McGettigan, 2018).

Al reconocer su importancia para el desarrollo regional, los diferentes gobiernos alrededor del mundo están introduciendo iniciativas políticas que promueven beneficios económicos para las microempresas y así generar más empleos. Como es el caso del gobierno de Australia que nombró su primer ministro de pequeñas empresas en 2013 y desde entonces han destinado una serie de políticas comprometidas con su crecimiento.

Según los informes realizados sobre el impacto de las reformas COAG: Regulación de Negocios y EFP, publicadas en mayo de 2012, en promedio los beneficios generados por las reformas son de $\$ 1.05 \mathrm{mil}$ millones por año (Koplitz, Nolte, Pouliot, Vukovich y Beidler, 2018).

A pesar de las iniciativas políticas que estimulan el crecimiento de las microempresas, éstas aún se enfrentan con varias dificultades al encontrarse en el final del triángulo del valor corporativo, son los primeros en recibir el impacto en las variaciones del mercado. Es decir, pueden sufrir crisis económicas por la pérdida imprevista de ingresos, recorte de personal y alta exigencia de servicio. Además de la carencia económica, la falta de preparación del personal administrativo opera como factor de riesgo en el desarrollo de estrategias para el control en crisis o incertidumbre del mercado fluctuante. Adicional a ello, las microempresas al ofrecer servicios especializados, deberán renovar sus estrategias actuales para entrar en el mercado, por lo cual es necesario transformar las oportunidades de crecimiento conforme a las demandas de servicio puesto que estas varían. Cabe mencionar que las herramientas tecnológicas tales como las redes sociales son instrumentos que favorecen la promoción de servicios y reducción de gastos (Binder, Buglass, Betts y Underwood, 2017).

\section{Inversión extranjera como herramienta del de- sarrollo regional}

Para este caso en particular, abordaremos la inversión extranjera como respuesta frente a la problemática 
del desempleo, puesto que la relación entre el crecimiento económico regional y la inversión extranjera es un elemento clave para aumentar el producto interno bruto, lográndose a través de la promoción del desarrollo industrial, competitividad y creación de nuevos empleos, igualmente, estos factores pueden apoyarse de la tecnología para tener mayor acceso al mercado global.

El desarrollo regional es un proceso que involucra la integración de un complejo sistema de regiones; en el cual se incorporan elementos sociales, económicos y culturales con el objetivo de enriquecer el índice económico a través del desarrollo territorial y ambiental. Sin embargo, la integración de las diferencias regionales es considerada actualmente como una limitación; es necesario poner en práctica actividades que promuevan la diversidad cultural para conciliar el desarrollo y disminuir la desigualdad.

Es responsabilidad del estado detectar las causas de la desigualdad regional e intervenir la problemática a través de políticas públicas.

Asociarse con compañías extranjeras conduce al crecimiento económico en las actividades comerciales de las empresas locales, en otras palabras, permite la integración financiera entre los países en desarrollo y el mercado económico mundial; es un camino directo hacia la globalización e internacionalización. Normalmente el inversionista extranjero posee el $10 \%$ o más de las acciones de la empresa asociada, sin embargo, la inversión extranjera pareciera tener otros motivos respecto a la financiación internacional, puesto que al inversionista se le otorgan derechos de propiedad e influencia administrativa sobre el capital y control de las actividades económicas, así como la autoridad para ordenar cambios en la estructura organizacional (Chang, DeVore, Granger, Eapen, Ariely y Hernandez, 2017).

Los efectos de la inversión extranjera equilibran el costo-beneficio en el desarrollo de las empresas regionales; por ejemplo, disponer de un equipo administrativo con formación financiera y experiencia en el mercado internacional incrementa la seguridad de las inversiones, debido a que los inversionistas ejecutan análisis minuciosos sobre la seguridad económica, seguridad política, posición geográfica y proporción del mercado. Estos elementos de análisis varían según el tipo o sector de inversión para obtener mejores ganancias. La integración de la economía de escala en la producción (estructura y preferencia del mercado) y la búsqueda de recursos (materia prima, infraestructura) son los factores determinantes que motivan la inversión extranjera, y dependen de elementos o condiciones del país que varían según contexto económico, político, social y tecnológico (Galizzi y Whitmarsh, 2019).

\section{Fenómeno de la globalización como reemplazo de la economía tradicional}

En nuestros días, no es un secreto que la globalización ha dominado el sistema capitalista contemporáneo; entendiendo este como un proceso social e históricamente complejo que se ha venido desarrollando durante siglos.

En la actualidad, se cuestiona si las economías tradicionales socialistas, que generalmente son típicas de los países subdesarrollados, pueden dar o no respuesta a la necesidad del mercado económico moderno, (Fanghella, d'Adda y Tavoni, 2019).

En 2016, los resultados del índice de globalización (KOF) permitieron relacionar la competitividad global con el producto interno bruto entre los países más globalizados (los países más globalizados fueron Holanda, seguido de Irlanda, Bélgica, Austria, Suiza, Singapur, Dinamarca, Suecia, Hungría, Canadá, Finlandia y Portugal. Los países en transición fueron: Hungría, Eslovaquia, República Checa, Polonia, Lituania, Bulgaria, Eslovenia, Croacia, Rumania, Serbia, Bosnia y Herzegovina, Montenegro y Macedonia). Como resultado, los países que se involucraron con el proceso de transición de su sistema económico tradicional, lograron un crecimiento económico considerable, por lo que se concluyó que la globalización no es un impedimento para el crecimiento de la economía y el desarrollo en general. Así mismo, Corea del Sur, Hong Kong y Taiwán recientemente realizaron su proceso de transición hacia la industrialización, logrando aproximarse al nivel de desarrollo económico de los países altamente desarrollados (Ghesla, Grieder y Schmitz, 2019).

Sin embargo, es necesario aclarar que los países en transición deben generar estrategias para una mejor posición entre los países más desarrollados, el progreso económico de estos países aún está por debajo de los objetivos de la modernización y como consecuencia, la globalización no puede consolidarse; puesto que los países en donde no se ha conseguido aminorar la pobreza no pueden asegurar una estabilidad económica, aunque la consolidación también puede estar sujeta a las fluctuaciones del mercado. A pesar de las restricciones económicas, mientras haya cooperación entre las regiones y las reformas especiales sean estimuladas para acelerar el proceso 
de transición, la problemática podría ser intervenida con optimismo en lugar de ser considerada como una limitación (Martinetti, Caughron, Berman, André y Sokolowski, 2019).

No obstante, si comparamos la realidad de estos países con la promesa de la globalización, encontraríamos que la mayoría de los países en transición confrontan problemas significativos en relación al endeudamiento internacional, presupuesto desequilibrado, tasas elevadas de desempleo y procesos insuficientes para la reestructuración de las reformas. En otras palabras, la oferta del crecimiento para el sistema económico que proponen los países más desarrollados aún no ha dado resultados proporcionales. Por esta razón, algunos críticos de la globalización plantean que su esencia es egoísta y ambiciosa y beneficia a los más desarrollados. Cabe señalar que estos países han establecido estrategias para el desarrollo y reformas políticas a largo plazo, pero los países en transición no pueden proyectarse sin el apoyo financiero externo. Sin duda, el capitalismo liberal es el actual ganador de la futura organización mundial (Chien y Chi, 2019).

Se considera que los países en transición tienen la responsabilidad de enfocarse en un nuevo paradigma económico, en donde deberán priorizar el concepto de la nueva economía, la reestructuración institucional, participación moderna del estado, desindustrialización y nuevas políticas industriales. Esto quiere decir que los países en transición tienen como desafío encaminarse hacia nuevos paradigmas económicos para su desarrollo y participación internacional (Lonial y Raju, 2015).

\section{Las redes sociales en el contexto de desarrollo global}

Las redes sociales se han definido como instrumentos de interacción social a través de los cuales pueden realizarse actividades económicas, políticas, académicas, entre otras que han permitido comunicar al mundo entre sí, incluso, hasta reemplazar los medios de comunicación tradicionales como la televisión, el periódico o los programas de radio.

El creciente potencial de las redes sociales es considerado objeto de estudio a nivel global, principalmente en los países en desarrollo donde el uso de sus recursos es considerablemente inferior en comparación con los países más desarrollados; esto se debe a diversos factores, entre ellos, la falta de acceso por desigualdad social. Aunque estas limitaciones también se relacionan con los componentes políticos, ambientales y culturales, que como sabemos, interactúan significativamente con las redes sociales, como consecuencia de ello, las empresas pueden dirigir estrategias comerciales en función de satisfacer las demandas de los usuarios y contribuir a su propio desarrollo.

Si bien las observaciones y percepciones individuales entre los sujetos son fuentes de información útil en términos de estadísticas de consumo, se ha demostrado la necesidad de establecer un modelo interactivo, en otras palabras, redes sociales. Esta redirección frente las necesidades del mercado, es considerada de las mayores contribuciones de las redes sociales a los sistemas económicos actuales (Ashraf, Camerer y Loewenstein, 2005).

Este sistema interactivo ha establecido nuevos espacios en las plataformas digitales en donde las personas del común pueden intercomunicarse con quienes admiran o con personas famosas en general. El efecto de esta interacción se ha convertido en la principal estrategia comercial de las empresas; conocido como marketing digital. Aunque la influencia de las celebridades está condicionada por el vínculo de empatía, credibilidad y fidelidad que establezcan con su audiencia a través de la exposición de su vida personal, laboral o académica. Una vez esta se construye, se utiliza como mecanismo para transformar las actitudes y preferencias de los usuarios. Sin embargo, la configuración del uso actual del marketing se ha utilizado entorno a diversas intenciones, tomando como ejemplo, la promoción de campañas sociales que estimulan un grupo significativo de personas para intervenir problemáticas sociales (Baca-Motes, Brown, Gneezy, Keenan y Nelson, 2013).

La publicidad social es el mecanismo más efectivo para establecer vínculos con la realidad o problemáticas sociales y tiene por objetivo, generar beneficios públicos a largo plazo, en donde la participación y toma de conciencia por parte de la comunidad, es fundamental. A través de la difusión de campañas informativas, más conocidas como comunicación de marketing, se ha logrado reducir significativamente la actitud social indiferente, transformándola en la intención participativa en relación a las problemáticas sociales presentes. En este orden de ideas, las redes sociales han impulsado campañas benéficas en donde la identificación con las celebridades ha llevado a los usuarios a realizar diferentes tipos de donaciones, es innegable la influencia de dichas celebridades sobre la toma de decisiones económicas y participativas de las personas sin ningún reparo, 
lo que puede generar incluso información distorsionada o poco provechosa. Esto quiere decir que, si bien el marketing comercial contribuye al desarrollo económico y social, también es un fenómeno inevitablemente retrógrado en términos del progreso de información auténtica, en donde se obtiene como resultado, la intencionalidad de los mensajes alterados (Baca-Motes y cols., 2013).

A pesar de ello, las virtudes del crecimiento de las redes sociales han influenciado rápidamente el desarrollo de novedosos tipos de marketing y de comunicación en general, como las estrategias modernas de comunicación e interacción política. Como resultado, las plataformas virtuales han creado espacios para la participación de la opinión pública, que tienen la finalidad de permitirles a los participantes calificar o valorar a los candidatos, partidos y políticas públicas. Esta herramienta se conoce generalmente como marketing político. Esto quiere decir que, las redes sociales tienen una responsabilidad significativa en las épocas de campaña electoral. Además, permiten seleccionar una población específica a la cuál dirigir su publicidad en donde la intención principal es adquirir nuevos votantes. Por ejemplo, los expertos en marketing de las campañas presidenciales en Estados Unidos, a través de los beneficios de las redes sociales lograron posicionar en la presidencia a sus candidatos, en 2008 a Barack Obama y en 2017 a Donald Trump. Entre las estrategias empleadas, se utilizaron las opiniones de los votantes para hacer ajustes atractivos en su discurso político, orientados hacia las necesidades actuales de los usuarios (Ashraf y cols., 2005).

Las plataformas digitales que actualmente dominan el marketing político son Facebook, Twitter, Instagram y YouTube. Sin embargo, en el caso particular de la campaña presidencial de Donal Trump, la estrategia de su equipo estuvo enfocada concretamente en Facebook y Twitter, que finalmente son las redes sociales con más potencia. Allí, obtuvo una ventaja significativa de seguidores en contraste con su oponente Hilary Clinton, quien optó por abordar un número mayor de redes sociales con la intención de llegar a más personas. Según los resultados electorales, no fue necesario que la campaña de Donal Trump se expandiera a todas las redes sociales para ganar las elecciones, por lo que podríamos concluir que la ventaja principal de las plataformas digitales consiste en poder dirigir un mensaje específico a una población seleccionada intencionalmente (Lonial y cols., 2015).

Adicional a ello, el recurso de la influencia de las personas famosas también es usado en el marketing político digital, en este caso las cualidades de las celebridades son medidas para diseñar estrategias más específicas en función de su audiencia en particular, aunque actualmente no existen instrumentos que evalúen condiciones particulares entre una celebridad y una marca.

Por su parte, Las expresiones faciales son consideradas por una gran cantidad de estudios como la representación más exacta de la experiencia de los individuos, por lo que en el marketing digital se utiliza como herramienta de medición para las emociones el software Emotion ID que se encarga de analizar las expresiones faciales como reacción a un anuncio o video publicitario.

Por otro lado, el concepto de tecnologías sociales se ha instaurado en las últimas décadas como consecuencia del vigor de la dimensión social en las ciencias tecnológicas, debido a que las ciencias sociales y las humanidades son quienes constituyen mayor volumen de investigaciones sociales. Ciertamente, algunos autores confirman en sus investigaciones que el aumento en el alcance y capacidad de la Web como mecanismo de interacción ha consolidado el desarrollo potencial de las tecnologías sociales; en donde finalmente se producen nuevas formas de relaciones y dinámicas de grupo ingeniosas entre los usuarios. Estas nuevas formas de comunicación han implicado que algunos sistemas de comunicación tradicionales sean reemplazados; por ejemplo, el correo electrónico y la mensajería (Ghesla y cols., 2019).

Anteriormente, había sido usado el término de arte social para referirse a los procesos mediante los cuales se podrían producir mejoras en la sociedad, no obstante, en la comprensión actual de tecnología social, se incluyen otros aspectos relevantes, como la interacción de conocimientos y la toma de decisiones. Las tecnologías sociales podrían ser definidas como cualquier tipo de tecnología usada para alcanzar algún objetivo en común de base social; entre las cuales se incluyen las herramientas de los medios de comunicación tradicional, la comunicación a través de un ordenador y las redes sociales. Por otro lado, las tecnologías sociales como tecnología digital se han empleado por los usuarios no solo para interactuar socialmente, si no para crear, perfeccionar e intercambiar contenido.

Las características principales de las tecnologías sociales consisten en: proporcionar la posibilidad de crear, añadir, editar contenido y facilitar el acceso distribuido para hacer uso de contenidos y comunicaciones. 
Las tecnologías sociales comprenden una cantidad significativa de herramientas tecnológicas que pueden ser empleadas por personas e instituciones del área pública o privada, incluso, pueden ser utilizadas simplemente como un instrumento de interacción entre ellas; estas herramientas abarcan tecnologías calificadas como redes sociales, la web 3.0 y herramientas de colaboración (Chaubal y cols., 2017).

El alcance de la interacción social mediante las tecnologías es un recurso potencialmente importante para ordenar el conocimiento de forma eficiente en diferentes contextos, en la cultura, economía e incluso, en el poder político. Diversas investigaciones han corroborado que el uso oportuno de las tecnologías sociales en actividades comerciales o profesionales han obtenido como resultado un aumento considerable en los objetivos propuestos (Koplitz y cols., 2018).

La integración de la tecnología social en la cotidianidad de los individuos podría convertirse en una necesidad, si las tendencias actuales prevalecen en el futuro.

En consecuencia, se puede concluir que la funcionalidad de la tecnología social actualmente está relacionada con propósitos sociales con la ayuda de los medios digitales. Es decir, las tecnologías sociales se comprenden como herramientas de información y comunicación que tienen relación con diferentes procesos sociales, económicos y culturales; además de elementos como ordenadores, teléfonos móviles y redes sociales.

Si bien el alcance de las redes sociales suministra información básica de manera adaptativa y conducida por los consumidores, en su mayoría, esas tecnologías tienen limitaciones que pueden cómodamente malinterpretarse, puesto que, al no establecerse comunicación no verbal, no es posible proveer la misma calidad de la interacción física y, por lo tanto, los procesos de intercambio de conocimientos y la habilidad para reconocer y resolver problemas, pueden verse alterados. Por otra parte, es posible que las viejas generaciones sean un poco incrédulas con respecto a las tecnologías sociales, de modo que es esencial implementar estrategias hábiles para instruir a la comunidad sobre el uso de las redes sociales. Habría que decir también, que la colaboración en línea actualmente no es la mejor propuesta para reemplazar las interacciones cara a cara que generalmente son necesarias para producir avances genuinos. (Baric y cols., 2018).
Las tecnologías sociales han permitido que sus usuarios se conviertan en participantes activos para los procesos de elección y creación del contenido, en lugar de únicamente consumirlo. Sin embargo, la naturaleza del contenido creado por el usuario generalmente varía en términos de calidad, desde extraordinarios trabajos de periodismo hasta spam o en algunas ocasiones, abuso.

Hasta ahora, hemos abordado el concepto de la globalización en términos del desarrollo económico y en relación a las tecnologías sociales; hemos visto cómo a través del crecimiento y la implementación de nuevas tecnologías, las industrias han puesto en práctica estrategias innovadoras para consolidarse en el mercado internacional actual, y cómo el desarrollo de nuevas tecnologías, han participado en la implementación de herramientas de comunicación social impactando potencialmente las sociedades alrededor del mundo, lo que finalmente sirve como referencia para los países en desarrollo. Ahora bien, exploraremos el fenómeno de la globalización desde una perspectiva de impacto social, pues los efectos de la globalización están dividas en dos opiniones controversiales. Por un lado, están quienes defienden el impacto positivo de la globalización en términos de progreso; es decir, se considera como un instrumento que ha estimulado el desarrollo económico en general a través de nuevas oportunidades laborales o espacios para la innovación. Por otra parte, se considera como un elemento que ha causado dificultades sociales; por ejemplo, pobreza, desempleo, marginación, inestabilidad económica, política, entre otros (Hagger y cols., 2018).

\section{Presión global sobre salarios y empleo}

Las compañías multinacionales toman decisiones para emplear estrategias de producción con calidad, mano de obra, herramientas, técnicas y beneficios estructurales en general, con el objetivo de disminuir impuestos fundamentados hacia el bienestar y equilibrio político.

En función del condicionamiento laboral para reducir los costos, una gran parte de la producción manufacturera se ha desplazado hacia los países en desarrollo; como consecuencia, la tasa de desempleo ha aumentado significativamente en la mayoría de compañías manufactureras de los países industrializados, desencadenando también la necesidad para promover la seguridad social, específicamente la tensión social por extender el seguro de desempleo 
financiado por el gobierno. A pesar de esta problemática, los países afectados por la disminución en las tarifas e impuestos, fueron forzados a disminuir gastos particularmente en el sector social, como recortes de personal e inversiones modestas en torno a la seguridad social, por lo que se considera que la presión dirigida hacia las industrias ha traído como resultado la transformación del sistema de seguridad social internacional (Novack y cols., 2018).

Ahora bien, aunque la mayoría de las contribuciones de las investigaciones determinan que los efectos de la globalización en términos de costo y beneficio han promovido indiscutiblemente el desarrollo de la economía en general, la posición de las investigaciones que valoran la globalización en la pobreza es, en cambio, controversial. En su mayoría, los estudios más destacados establecen que la globalización ha disminuido el grado de pobreza, sin embargo, análisis rigurosos determinan que los resultados de esas investigaciones son conceptualmente deficientes; en 2002, la confederación internacional Oxfam realizó un estudio sobre las normas e instituciones comerciales, con el cual se comprobó que estas instituciones operan continuamente a favor de los países desarrollados; además, ha demostrado la existencia del impacto negativo y positivo de la globalización en la pobreza. Dicha investigación a través de indicadores individuales de apertura comercial y financiera indagó de qué manera la globalización impactaba a los pobres en los países en desarrollo, con el objetivo de corroborar si hay probabilidad de una relación lineal o no lineal entre las variables globalización y pobreza. Sus resultados, como la mayoría revisados, presentan en gran medida que los países menos desarrollados han sido marginados en el proceso de la globalización en los últimos 15 años (Brown, Neath y Chater, 2007).

En 2001, el instituto mundial para la investigación de la economía del desarrollo (CIEM), realizó una investigación con el objetivo de analizar las tendencias de la desigualdad en 73 países que representan más de cuatro quintas partes de la población mundial y el PBI. Los resultados indican que en los últimos 20 años la desigualdad aumentó en dos tercios de estos 73 países; aunque el patrón no es consistente, evidencia un claro distanciamiento de las tendencias registradas al final de la segunda guerra mundial. Respecto a la tecnología y el comercio, señalan que no son fundamentalmente los elementos más importantes para incrementar la desigualdad del ingreso en los países en desarrollo. Sin embargo, consideran la globalización y la tecnología como componentes relevantes que explican el crecimiento de la desigualRevista Innovación Digital y Desarrollo Sostenible dad en los últimos 20 años en los países en desarrollo. Finalmente, determinan que los elementos más importantes para los países en desarrollo son las normas sociales, el salario y las situaciones macroeconómicas.

Algunas investigaciones han explorado los efectos de la globalización en relación a la inseguridad global, como la carencia de garantías sociales, inestabilidad alimentaria, temor hacia actos terroristas, entre otros. El análisis de estas investigaciones ha establecido que la globalización ha incrementado la inseguridad económica, social, política y finalmente la sostenibilidad del progreso de la globalización (Brown y cols., 2007).

\section{METODOLOGÍA}

El caso de esta investigación en particular se basa en la descripción genérica de elementos que han sido trascendentales en la globalización. La selección, organización y análisis del material bibliográfico retomado para este artículo se fundamenta en el enfoque cualitativo, apoyado en el tipo de investigación documental. En la selección del material bibliográfico se identificaron un total de 90 textos producto de las búsquedas en bases de datos académicas tales como: JCR (Journal Citation Reports), Scopus, DICE (Difusión y Calidad Editorial de las Revistas Españolas de Humanidades y Ciencias Sociales y Jurídicas), ISOC, Iresie, Google Académico, Redalyc, Scielo, EBSCO y Scopus. En la selección de los documentos se tuvieron en cuenta criterios básico como: referentes normativos, políticos y programáticos adoptados por Colombia; hallazgos de investigaciones en el ámbito internacional y nacional; periodo de la publicación (1972-2019).

Para la organización y análisis del material, se elaboró una lista de categorías en la cual se consignaron datos como:, autor, año, referencia bibliográfica y país de publicación, conceptos básicos (globalización, migración, teléfono móvil, redes sociales, empleo, salario,...), características de las herramientas implementadas en el marco de las políticas de bilingüismo en Colombia; elementos relacionados con los aciertos, desaciertos, obstáculos y especificidades del contextos en el que se aplican dichas políticas. Como metodología se implementaron elementos propios de un análisis documental el cual según Van Dijk (1995), consiste en implementar estructuras discursivas y significados, así como algunos aspectos u opiniones generales, teniendo en cuenta elemen- 
tos psicológicos, cognitivos, contextuales e ideológicos, de ahí su complejidad interdisciplinaria.

\section{DISCUSIÓN}

Las propuestas nacionales en general cambian de un país a otro debido a que los distintos países se encuentran en periodos de desarrollo diferentes y por lo tanto sus prioridades también, sin embargo, algunos procesos de política nacional son generales en todos los gobiernos; por ejemplo, la inversión en la educación y capacitación, asimilación de normas laborales básicas, suministro y mejoría de la protección social, la lucha en contra de la desigualdad nacional y habilidades para debatir sobre la globalización. En contraste, a raíz de un mundo cada vez más globalizado algunas otras políticas han tomado importancia, como las políticas macroeconómicas sostenibles, políticas que estimulan un clima de inversión sólido y la organización financiera moderada.

Actualmente existe un gran consenso respecto a la importancia de la educación y capacitación como elementos que deben ser integrados en las acciones políticas, como también las entidades educativas que promueven la innovación. Comúnmente se sugiere para los países en desarrollo que conduzcan acciones en función del suministro de educación básica gratuita, al igual que los servicios básicos de salud. Si bien la globalización ha permitido reorientar las acciones políticas en función de estrategias innovadoras para el desarrollo en general, también ha implicado un numeroso desempleo estructural, por lo cual es importante dirigir capacitaciones hacia los desempleados con el fin de trasladarse a ocupaciones de mayor demanda (McCollough, 2000).

Hasta ahora existe una conciliación global respecto a que la incorporación de las cuatro normas laborales básicas hace de la globalización un proceso más equitativo, que son; la promoción de la no discriminación en el empleo, abolición del trabajo infantil, anulación del trabajo forzoso y negociación colectiva. No obstante, las diferencias entre las definiciones nacionales acerca de lo que verdaderamente significa trabajo infantil, trabajo forzoso y demás, deben ser respetadas.
Las investigaciones realizadas respecto al efecto de la globalización y el trabajo infantil están divididas en tres grupos; por una parte, algunos autores han advertido sobre los efectos negativos en países e industrias específicas en contraste con quienes se han interesado en la utilidad de la legislación en algunos países más desarrollados, y finalmente, las publicaciones que evidencian el efecto de la globalización en el trabajo infantil. Actualmente es evidente el incremento del trabajo infantil como consecuencia de los alcances de la globalización; el incremento de las empresas manufactureras ha ampliado las oportunidades laborales para mujeres y niños que han dado origen a diversas empresas familiares. Algunos de los beneficios de la globalización han sido causados en gran medida por la transformación del empleo informal hacia contratación y salarios de acuerdo con las leyes federales del trabajo. Por otro lado, aunque la posibilidad del trabajo infantil ha beneficiado esencialmente los ingresos de las familias de niveles socioeconómicos bajos, los países más desarrollados por efecto de la presión pública, han implementado leyes impeditivas respecto al curso del trabajo infantil, seguidos por las multinacionales que comenzaron a restringir la participación infantil en sus procesos laborales. Como consecuencia, las investigaciones recientes afirman que la globalización ha disminuido el trabajo infantil a nivel formal; en otras palabras, ha luchado en contra de la explotación infantil (Carter y Irons, 1991).

En contraste con la posición de explotación laboral respecto a la formalización del trabajo infantil, el aumento de las oportunidades laborales para las mujeres es considerado como un proceso contribuyente para su lucha por la libertad e independencia económica; aunque las investigaciones iniciales concluyen que la globalización contribuye a la desigualdad de género. Sin embargo, la exclusión laboral en contra de la mujer continúa extendiéndose en la actualidad. Respecto a las mujeres todavía no adquieren poder económico a pesar del crecimiento en tanto a oportunidades laborales. En realidad, las mujeres acceden a propuestas en donde consienten recibir salarios inferiores con la intención de compensar la responsabilidad familiar con el empleo. Además, una cantidad considerable de trabajadoras no cuentan con estrategias efectivas en función de la distribución de su salario y la responsabilidad doméstica no remunerada ha sido puesta tradicionalmente en la mujer, aún en los hogares en donde las mujeres proveen el único ingreso. Por su parte, se ha planteado una perspectiva en la que se comprende la global- 
ización como un fenómeno que indudablemente ha producido consecuencias económicas perjudiciales para las mujeres, no obstante, ha promovido e inspirado un grupo de mujeres trabajadoras y ordenadas, entendiéndose como un movimiento anti sistémico. Las políticas comerciales y las competencias individuales afectan en un modo diferente a los hombres y a las mujeres, y que las políticas de liberalización comercial tienen la responsabilidad de reestructurarse esencialmente en función del desarrollo social y la igualdad de género (Carter y cols., 1991).

\section{Globalización y migración}

En el análisis sobre el impacto de la globalización en la migración se observan niveles significativamente inferiores de migración internacional actual en comparación con las migraciones a gran escala en la historia mundial. En su mayoría, las investigaciones han concluido que esto se debe a las leyes selectivas de inmigración que han establecido los países industrializados. Actualmente los efectos de la globalización no favorecen al proceso de migración de las personas con competencias inferiores en relación a los migrantes altamente calificados. Esto quiere decir que la emigración ha perjudicado significativamente a los países en desarrollo, debido a que los migrantes pertenecen en su mayoría a la fracción más productiva y preparada del país y generalmente los mejores preparados que emigran provienen de países latinoamericanos cercanos a Estados Unidos, como República Dominicana, El salvador, Guatemala, Jamaica y México. Sin embargo, las investigaciones sobre los reportes del Banco Mundial del 2003 han concluido que la migración ha beneficiado a las empresas de envío en los países en desarrollo a causa de las remesas enviadas por los migrantes. A pesar de que estas remesas mejoran perceptivamente el nivel de vida de las familias destinarias, la emigración de estos profesionales no estimulan el desarrollo del país de origen, puesto que la emigración de estos trabajadores continúa representando un problema, fundamentalmente para los países en desarrollo (Epley, Waytz y Cacioppo, 2007).

En su mayoría, el contenido explorado permite promover propuestas de intervención estructural por parte de los gobiernos nacionales e internacionales quienes tienen la responsabilidad de aliviar las problemáticas desencadenadas a causa de la globalización. Como consecuencia del acuerdo en la opinión sobre la evidente demanda de participación por parte de los gobiernos a raíz de la globalización, actualmente existen numerosas investigaciones que proponen intervenciones políticas en función de combinar la cooperación del estado y las políticas, aunque diferentes investigaciones han concluido que la globalización ha disminuido la autonomía del estado frente a los asuntos económicos y sociales (Epley y cols., 2007).

\section{CONCLUSIONES}

La mayoría de las propuestas de acción política solo podrían ejecutarse eficazmente si se lleva a cabo la cooperación internacional, sin embargo, es importante considerar que algunos países no están conectados con el sistema de globalización actual, lo que cual no favorece cuando se requiere un apoyo económico sustancial.

Se considera importante realizar las propuestas sobre el suministro de mayor y mejor apoyo extranjero, alivio de la deuda externa para los países en desarrollo más endeudados y recomendaciones en relación a los asuntos ambientales, como el calentamiento global. Por último, si las limitaciones en las acciones políticas dependen de los vacíos en el sistema de gobernanza actual, seguramente es necesario dar origen a nuevas instituciones internacionales.

Los elementos económicos centrales en el progreso de la globalización pueden sintetizarse en los siguientes aspectos: por un lado, pese a que la participación de los países en desarrollo en el mercado mundial ha reducido significativamente, el comercio internacional ha aumentado en más del triple; por otra parte, aunque la participación de los países de bajos recursos en la inversión extranjera directa mundial ha reducido preocupantemente, la Inversión Extranjera Directa - IED, en todo el mundo ha incrementado más de 20 veces; por consiguiente, la inversión de cartera a nivel global se ha extendido más de 20 veces, aun cuando la participación de los países de bajos ingresos se aproxima a cero, y la fragmentación de la producción, en su mayoría, está relacionada con la reducción de la cooperación de los países en desarrollo en la producción internacional.

El efecto social de la globalización en la pobreza, el trabajo infantil, el género y la migración, han expuesto ciertamente una constante controversia. Si bien existe aparentemente un acuerdo global en el que se considera que la globalización ha generado mayores ganancias en lugar de costos, adicionalmente, 
que ha opacado las divergencias nacionales entre los países a través de la experiencia particular y nacional; y también que ha incrementado la inseguridad económica y política. Establecer vínculos causales entre las variaciones de la pobreza y desigualdad con el incremento de la globalización continúa siendo un desafío, puesto que los alcances de la globalización van mucho más allá de sus características económicas; por ejemplo, la creciente influencia de la globalización en la salud mundial y las crisis ambientales. No obstante, aunque la globalización no sea la causa fundamental en la desigualdad de ingresos y la pobreza, es factible que haya asistido al bajo nivel de rendimiento de los esfuerzos para disminuir la pobreza.

Para finalizar. Aunque generalmente la globalización no ha concluido los beneficios esperados, el origen del problema consiste en que el proceso de la globalización debería ser dirigido de la manera correcta en lugar de analizar si éste puede ser un fenómeno que contribuye o no al bienestar de los pobres del mundo, aunque generalmente esto no ocurre. Como se ha detallado anteriormente, los procesos vinculados con la implementación y el financiamiento de políticas nacionales e internacionales para conducir el sistema de la globalización continúan siendo controversiales; sin embargo, hasta ahora se ha concluido que los gobiernos tienen la responsabilidad de invertir en educación, capacitación y promoción de la innovación así como debe fortalecer las estrategias en contra de la desigualdad nacional y ofrecer espacio y oportunidades para discutir la globalización. Otro aspecto importante a nivel internacional se refiere a las acciones políticas que han recibido respaldo por las investigaciones contemporáneas, como la nueva arquitectura del sistema financiero y el crecimiento de las negociaciones comerciales. Aunque finalmente es necesario el respaldo de muchas más investigaciones a nivel nacional e internacional para convertir a la globalización en un proceso más amigable en términos sociales, puesto que la globalización por sí misma no favorece a la población que actualmente vive bajo condiciones económicas inadmisibles. 
Aaker, J. L. (1997). Dimensions of brand personality. Journal of Marketing Research, 34(3), 347-356.

Ashraf, N., Camerer, C., y Loewenstein, G. (2005). Behavioral Economist. Journal of Economic Perspectives, 19(3), 131-145.

Baca-Motes, K., Brown, A., Gneezy, A., Keenan, E. A., \& Nelson, L. D., (2013). Commitment and behavior change: Evidence from the field. Journal of Consumer Research, 39(5), 1070-1084.

Baines, D., Bates, I., Bader L., Hale, C., \& Schneider, P. (2018). Conceptualising production, productivity and technology in pharmacy practice: a novel framework for policy, education and research. Human Resources for Health, 16(1), 51-58.

Baric, V., Andreassen, M., Öhman, A., \& Hemmingsson H. (2018). Using an interactive digital calendar with mobile phone reminders by senior people - a focus group study. BMC Geriatrics. 19(1), 11-21.

Bauleo, MF., Van, Dijk F., \& Radon, K. (2018). One's Workplace, Other's Home? Work and Health of Domestic Workers in Argentina. Annals of global Health, 84(3), 450-458.

Binder, JF., Buglass, SL., Betts, LR., \& Underwood, JD. (2017). Online social network data as sociometric markers. The American Psychologist, 72(7), 668678. https://doi.org/ 10.1037/amp0000052.

Brown, G., Neath, I., y Chater, N. (2007). A temporal ratio model of memory. Psychological Review, 114, 539-576.https://doi.org.org/10.1037/0033295X.114.3.539.

Carter, JR., y Irons, MD. (1991). Are economists different, and if so, why? Journal of Economic Perspectives, $5(2), 171-177$

Chang, LL., DeVore, AD., Granger, BB., Eapen, ZJ., Ariely, D., \& Hernandez, AF. (2017). Leveraging Behavioral Economics to Improve Heart Failure Care and Outcomes. Circulation, 136(8), 765-772.https:// doi.org/10.1161/CIRCULATIONAHA.117.028380.

Chaubal, T., \& Bapat, R. (2017). Geographic Tongue. The American Journal of Medicine, 130(12), 533-534.https:doi.org/10.1016/j.amjmed.2017.06.016. Epub 2017 Jul 14

Chien, L., \& Chi, S. (2019). Corporate image as a mediator between service quality and customer satisfaction: difference across categorized exhibitors. Heliyon, 5(3),1317.https:doi.org/10.1016/j.amjmed.2017.06.016. Epub 2017 Jul 14.

Epley, N., Waytz, A., \& Cacioppo, JT. (2007). On seeing human: A three-factor theory of anthropomorphism. Psychological Review, 114(4), 864-886.ht-
Fanghella, V., d'Adda, G., \& Tavoni, M. (2019). On the Use of Nudges to Affect Spillovers in Environmental Behaviors. Frontiers in Psychology, 10, 61-69. https:doi.org/10.3389/fpsyg.2019.00061. eCollection 2019.

Galizzi, MM., \& Whitmarsh, L. (2019). How to Measure Behavioral Spillovers: A Methodological Review and Checklist. Frontiers in Psychology, 10, 342-347. https:doi.org/10.3389/fpsyg.2019.00342. eCollection 2019.

Ghesla, C., Grieder, M., \& Schmitz, J. (2019). Nudge for Good? Choice Defaults and Spillover Effects. Frontiers in Psychology, 10, 178-190.https://doi.org/ 10.3389/fpsyg.2019.00178. eCollection 2019.

Hagger, MS., \& Weed, M. (2018). Do interventions based on behavioral theory work in the real world? The international Journal of Behavioral Nutrition and Physical Activity, 16(1), 36-43.https//doi.org/ 10.1186/s12966-019-0795-4.

Koplitz, SN., Nolte, CG., Pouliot, GA., Vukovich, JM., \& Beidler, J. (2018). Influence of uncertainties in burned area estimates on modeled wildland fire PM2.5 and ozone pollution in the contiguous U.S. Atmospheric Environment, 191, 328-339.https:// doi.org/10.1016/j.atmosenv.2018.08.020.

Krupka, EL., \& Weber, R. (2013). Identifying social norms using coordination games: why does dictator game sharing vary? Journal of European Economic Association, 11(3), 495-524.

Lonial, S., \& Raju, PS. (2015). Impact of service attributes on customer satisfaction and loyalty in a healthcare context. Leadership Health Service, 28(2), 149166.https:doi.org/ 10.1108/LHS-12-2013-0045.

Martinetti, MP., Caughron, R., Berman, H., André, J., \& Sokolowski, MB. (2019). The Behavioral Economics of Alcohol Demand in French and American University Students. Alcoholism. Clinical and Experimental Research, 43(3), 531-544.

McCollough, MA. (2000). The effect of perceived justice and attribution regarding service failure and recovery. Journal of Hospitality and Tourism Research, 24(4), 423-447.https:doi.org/10.1111/ acer.13954. Epub 2019 Feb 7.

Novack, MN., Hong, E., Dixon, DR., \& Granpeesheh, D. (2018). An Evaluation of a Mobile Application Designed to Teach Receptive Language Skills to Children with Autism Spectrum Disorder. Behavior Analysis in Practice, 12(1), 66-77.https:doi.org/ 10.1007/s40617-018-00312-7. eCollection 2019 Mar.

O’Neill, MF., \& McGettigan, G. (2018). Spanish biotechnology: anyone for PYMEs? Drug Discovery 
Today, 10(16), 78-81.https:doi.org/ 10.1016/

S1359-6446(05)03549-X.

Raduege, T. J. (2018). Benefits and Services. Issue Brief (Health Police Trank Service), 24: 1-43.

Reid, J. R., \& Baker, R. S. (2018). Designing and testing an educational innovation. Pediatric Radiology, 48(10), 1406-1409.https:doi.org/10.1007/ s00247-018-4193-x.

Sis-Çelik, A., \& Kırca, N. (2018). Prevalence and risk factors for domestic violence against infertile women in a Turkish setting. European Journal of obstetrics, Gynecology, and reproductive Biology, 231, 111-116.https:doi.org/10.1016/j.ejogrb.2018.10.032. Epub 2018 Oct 13.

Slaughter, J. E., Zickar, M. J., Highhouse, S., \& Mohr, D. C. (2004). Personality trait inferences about organizations: Development of a measure and assessment of construct validity, Journal of Applied Psychology, 89(1), 85-103.htts:doi.org/ 10.1037/00219010.89.1.85.

Tang, S., Ghose, B., Hoque, M. R., Hao, G., \& Yaya, S. (2019). Women Using Mobile Phones for Health Communication Are More Likely to Use Prenatal and Postnatal Services in Bangladesh: Cross-Sectional Study. JMIR mHealth and uHealth, 7(2), 1015.https:doi.org/ 10.2196/10645. 